Full length article

\title{
Reliability of joint kinematic calculations based on direct kinematic and inverse kinematic models in obese children
}

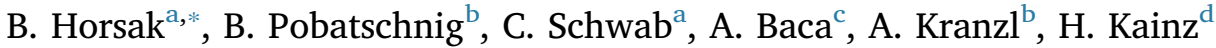 \\ a St. Pölten University of Applied Sciences, Institute of Health Sciences, St. Pölten, Austria \\ b Orthopaedic Hospital Vienna-Speising, Laboratory of Gait and Movement Analysis, Vienna, Austria \\ ${ }^{\mathrm{c}}$ University of Vienna, Department of Biomechanics, Kinesiology and Applied Computer Science, Vienna, Austria \\ ${ }^{\mathrm{d}}$ Human Movement Biomechanics Research Group, Department of Kinesiology, KU Leuven, Leuven, Belgium
}

\section{A R T I C L E I N F O}

\section{Keywords:}

Global optimization

Repeatability

Gait analysis

Test-retest

Musculoskeletal modelling

\begin{abstract}
A B S T R A C T
Background: In recent years, the reliability of inverse (IK) and direct kinematic (DK) models in gait analysis have been assessed intensively, but mainly for lean populations. However, obesity is a growing issue. So far, the sparse results available for the reliability of clinical gait analysis in obese populations are limited to direct kinematic models. Reliability error-margins for inverse kinematic models in obese populations have not been reported yet. Research questions: Is there a difference in the reliability of IK models compared with a DK model in obese children? Are there any differences in the joint kinematic output between IK and DK models?

Methods: A test-retest study was conducted using three-dimensional gait analysis data from two obese female and eight obese male participants from an earlier study. Data were analyzed using a DK model and two OpenSimbased IK models. Test-retest reliability was compared by calculating the Standard Error of Measurement (SEM) along with similar absolute reliability measures. A Friedman Test was used to assess whether there were any significant differences in the reliability between the models. Kinematic output of the models was compared by using Statistical Parametric Mapping (SPM).

Results: No significant differences were found in the reliability between the DK and IK models. The SPM analysis indicated several significant differences between both IK models and the DK approach. Most of these differences were continuous offsets.

Significance: Reliability values showed clinically acceptable error-margins and were comparable between all models. Therefore, our results support the careful use of IK models in overweight or obese populations, e.g. for musculoskeletal modelling studies. The inconsistent kinematic output can mainly be explained by different model conventions and anatomical segment coordinate frame definitions.
\end{abstract}

\section{Introduction}

Most conventional gait models use direct kinematics (DK) to calculate joint angles and their derivatives. DK calculate joint angles as Euler angles between adjacent segment reference frames. These are defined directly from the experimental markers, which are assumed to be rigidly attached to the bones [1]. DK gait models are limited to joint kinematic and kinetic analysis. In contrast to DK models, the more sophisticated musculoskeletal (MSK) models (e.g. OpenSim [2] or AnyBody [3]) use inverse kinematics (IK), also known as global optimization, to calculate joint angles. MSK models have the main advantage of enabling additional analyses such as muscle-tendon length and force estimation [4], induced acceleration analysis [5], and joint contact force calculations [6]. These analyses may help to identify causes for secondary pathologies in obese populations (e.g. joint degeneration) and increase our understanding of gait in people with obesity by adding information about gait strategies at the musculoskeletal level.

If clinicians or scientists use either kind of these models to aid decision-making in clinical practice, reliability is an essential issue. In order to serve as a valuable tool, e.g. to evaluate therapy progress, one has to know if an obtained result reflects a true difference or if this difference falls into the variability of the underlying measurement technique [7]. The reliability of DK models has been assessed extensively over the last years. Research certifies moderate to good reliability in lean adults, typically developing children, children with cerebral palsy, stroke patients as well as acceptable reliability in obese children [8,9]. The reliability of IK models has only been assessed in a limited number of studies including healthy adult participants, lean

\footnotetext{
* Corresponding author at: St. Pölten University of Applied Sciences, Institute of Health Sciences, Matthias Corvinus-Straße 15, A-3100, St. Pölten, Austria.

E-mail address: brian.horsak@fhstp.ac.at (B. Horsak).
} 
typically developing children, and children with cerebral palsy [10-12]. So far, no study has assessed the reliability of IK models to compute joint kinematics in obese children, even though MSK-IK models have already been used in this population to calculate muscle forces and joint-contact forces [13-15].

During the entire workflow of three-dimensional (3D) gait analysis, regardless of using IK or DK approaches, the same pitfalls exist in identifying anatomical landmarks, in placing markers accurately and reliably, as well as problems associated with soft-tissue displacement [16]. In an IK approach, an additional processing step is necessary, where the pose and marker positions of a generic model is adjusted to attain the best match with the experimental markers $[17,18]$. The quality of the results during this process is highly dependent on the experience of the examiner. This process gets even more challenging in an obese population due to large soft-tissue offsets. As a consequence, this step may add additional variability to the results. Therefore, it is of utmost importance to assess the reliability of IK models in estimating joint kinematics in obese children to build up confidence in the MSK simulation results for overweight and obese populations.

Accordingly, the primary aim of this study was to assess the reliability of two different IK models in obese children and to compare their reliability to the reliability of a DK model. A secondary aim was to identify if there are any differences in joint kinematics between the IK models and the DK approach. Based on the findings of previous IK and DK reliability studies in lean participants [8,10-12], we hypothesized that the reliability of gait kinematics in obese children will be similar between the IK and DK models. Based on earlier published data [19], we also hypothesized that there will be clinically relevant differences in joint kinematics between the IK and the DK models.

\section{Methods}

\subsection{Participants}

This study used the data from an earlier study [9]. The data comprised of a convenience sample of two females and eight males with an age-based body mass index (BMI) above the 97th percentile (mean \pm SD, age: $14.6 \pm 2.8$ years, height: $169.3 \pm 11.3 \mathrm{~cm}$, body mass: $99.2 \pm 21.7 \mathrm{~kg}$; BMI: $34.2 \pm 3.9 \mathrm{~kg} / \mathrm{m}^{2}$ ). Exclusion criteria were the existence of any syndromes associated with obesity (e.g. Prader-Willi syndrome), chronic joint disease, neuro-motor disease, or any history of a lower extremity joint surgery. Ethical approval was obtained by the local Ethics Committee (Ethics number: 1445/2013) prior to the study. Written informed consent was obtained from all participants and their legal representatives.

\subsection{Motion capturing}

All participants completed two gait analysis sessions, a test and a retest session, both separated by a minimum of one day and a maximum of seven days (on average $3 \pm 2$ days). The same assessor with one year of motion capture experience collected all gait analysis data. Participants walked barefoot on level ground at self-selected walking speed on a $12 \mathrm{~m}$ walkway until a minimum of five trials with no larger gaps in the marker trajectories for the left and right leg were recorded. The participants' walking speed was measured using photoelectric sensors. For the retest, only trials with walking speeds $\pm 5 \%$ from the baseline were used for subsequent analysis. The Cleveland Clinic marker set was used for motion capturing. This marker set comprises of twenty-seven retro-reflective spherical markers, some of which attached as clusters of three on rigid base plates to the thigh and shank, others to anatomical landmarks (Fig. 1). Motion capturing data were recorded using an eight-camera motion capture system (MX-series, Vicon, Oxford, UK) operated by the software Vicon Nexus (v.1.8.5) at $150 \mathrm{~Hz}$. Marker trajectories were filtered using a zero-lag 4th order lowpass Butterworth filter with a cut-off frequency of $12 \mathrm{~Hz}$. The cut-off frequency was determined a priori by residual analysis [20].

\subsection{Analyzed models}

The DK model (Cleveland Clinical marker set), which is well established in clinical practice [21-23], was created in BodyBuilder (Vicon Motion Systems, Oxford, UK). A previous pilot study confirmed acceptable intra- and inter-tester reliability for this marker set [23]. All joints in the DK model were ball-and-socket joints with three degreesof-freedom (DoF) with a rotation sequence of $y-x-z$ describing the flexion/extension, ab-/adduction, and internal/external rotation respectively. For estimating the hip joint center, the predictive approach described by Davis et al. [24] was used. To account for anterior soft tissue offsets of the anterior superior iliac spine (ASIS) markers, we placed the ASIS markers on the lateral side of the pelvis, so that the marker center reflects the anterior surface of the palpated ASIS landmark (from a sagittal point of view). The inter-ASIS distance was then measured manually by a spreading caliper and was input to the Davis regression equation. The pelvic angles were calculated from the pelvic coordinate system relative to the global coordinate system following the guidelines of $\mathrm{Wu}$ et al. [25].

Two different IK models were used. The 3-1-1-IK model was the 'gait2392' OpenSim model [26], a common used IK model, which includes three rotational DoF at the hip joint, one rotational DoF at the knee joint, and two separate rotational DoF for the talocrural and subtalar joints. The subtalar joint was locked due to an insufficient number of foot markers to track both DoFs. The second IK model (3-3-1IK model) was the same as the 3-1-1-IK model but allowed knee ab-/ adduction and internal/external rotation additionally to the flexion/ extension DoF. The 3-3-1-IK model was created to have an IK model with similar DoF as our DK model. Both generic IK models were scaled to each participant's anthropometry using scale factors calculated from the experimental marker positions and estimated joint centers [27]. During the IK task, only the pelvis, foot and cluster markers on the thigh and shank segments were tracked to calculate joint kinematics. Scaling and IK were performed in OpenSim 3.3 [2].

\subsection{Data reduction and analysis}

Except for marker reconstruction and kinematic modeling (IK and DK), all processing steps were performed in Matlab (v. R2017a, The Mathworks, Natick, MA). All data were time-normalized to $100 \%$ gait cycle (\%gc). All analyses were conducted for both body sides separately. The findings for the right side will be reported in the main manuscript, results for the left side are available as supplementary material. From the five trials available per participant, a mean representative trial was calculated and used for further analysis.

To assess the reliability of our models, which was our primary aim, key kinematic parameters comprising the minimum, maximum and the range of motion (ROM) from each kinematic waveform for all joints and planes were calculated for each model and for the test and retest session. The standard error of measurement (SEM) was used to assess the absolute test-retest reliability for the key kinematic parameters. The SEM was calculated as the square root of the mean square error term from a two-way repeated measures analysis of variance [28]. The total variability between the test and retest for the key kinematic parameters (RMSDp) and for the entire waveforms (RMSDw) were assessed by calculating the root mean square differences similar to Horsak et al. [29]. In addition, the mean and standard deviation (SD) of each parameter for both test sessions as well as the difference and the corresponding 95\% confidence intervals (CI) were calculated. An initial Shapiro-Wilk test indicated that the SEM and RMSDp variables were not normally distributed. Therefore, a Friedman test was used to determine if there were any significant differences in the SEM and RMSDp (across all key kinematic parameters, joints and planes, see Table 1) between the three models. Alpha level was set a priori to 0.05 .

For the comparison of joint kinematics between our models, which was our second aim, we used Statistical Parametric Mapping (SPM) and 


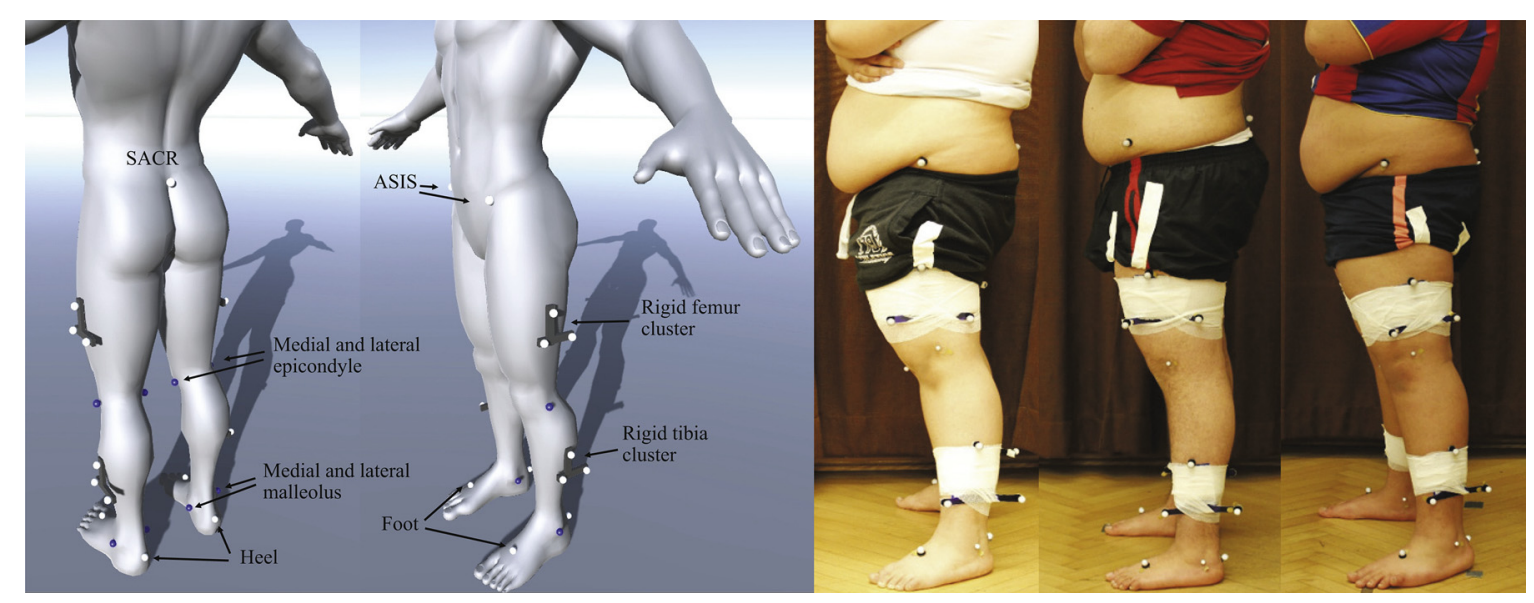

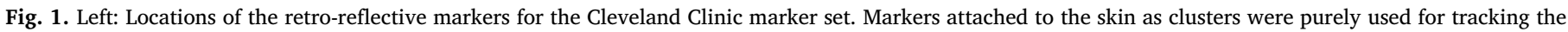

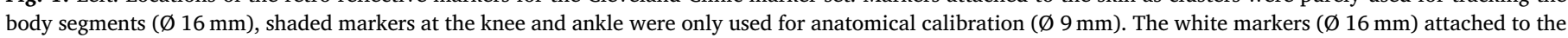

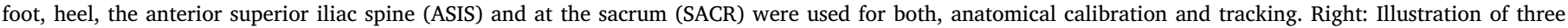
exemplary participants with attached markers. Reprinted with permission from Elsevier [29].

the Random Field Theory (RFT) provided by Pataky [30] within the SPM1D package available for Matlab (v.0.4, http://www.spm1d.org/). This method uses the entire one-dimensional (1D) continuous kinematic waveforms and the RFT to make probabilistic conclusions based on the random behavior of the $1 \mathrm{D}$ observational unit. It follows the same notion and logic as classical inference testing. We used paired $t$-tests to compare each waveform from the first test session between the DK model and each IK model. To account for multiple testing, the alpha level was Bonferroni corrected to 0.005 for the 3-3-1-IK vs. DK comparisons (alpha $=p / 10$ ) and to 0.006 for the 3-1-1-IK vs DK comparisons (alpha $=p / 8$ ). Normal distribution was initially checked using the corresponding normality test provided by SPM1D. If normality could not be assumed, the nonparametric version was used. The output of SPM provides SPM $\{\mathrm{t}\}$ values for each point of the investigated kinematic time series, and the threshold corresponding to the set alpha level. The SPM $\{\mathrm{t}\}$ values exceeding this threshold (marked as black bars below each figure, e.g. in Fig. 3) indicate significant differences in the corresponding portion of the time series.

\section{Results}

Reliability was similar between the DK and both IK models. The Friedman test did not indicate significant differences in the SEM $\left(\chi^{2}\right.$ $(2)=1.742, p=0.419)$ and $\operatorname{RMSDp}\left(\chi^{2}(2)=1.826, p=0.401\right)$ parameters between the analyzed models. The SEM, averaged across the min, max and ROM, was below $3^{\circ}$ (range: $1.6-3.0^{\circ}$ ) for all analyzed models in the sagittal and frontal plane and below $5^{\circ}$ for the transversal plane $\left(2.1-4.9^{\circ}\right)$. Results for the RMSDp and RMSDw showed a similar pattern for all three planes, but slightly higher mean values ranging from 2.2 to $5.4^{\circ}$ (Fig. 2 and Table 1). The left body side (see Supplementary material, Fig. S1 and Table S1) showed comparable reliability patterns, but slightly smaller values especially for the transversal plane in all models.

The comparison of joint kinematics between the DK and IK models showed several significant differences (Fig. 3). Compared to the DK models, the 3-3-1-IK model showed a continuous offset during the entire gait cycle for the sagittal pelvic and hip kinematics, differences in the frontal pelvic kinematics as well as differences during initial contact and late swing in the frontal hip kinematics. A small difference was also present for the maximum knee flexion during $20-30 \%$ of the gait cycle along with an almost continuous offset across the entire gait cycle for the transversal knee and the sagittal ankle kinematics. The 3-1-1-IK model showed a very similar pattern with additional differences in hip angles in the transversal plane compared with the DK model. The results of the left body side were similar and are reported in the Supplementary material (Fig. S2).

\section{Discussion}

The primary aim of this study was to evaluate the test-retest reliability of two different IK models and to compare their results to a DK approach in a sample of young obese children and adolescents.

All three models were found to display a similar and clinically acceptable level of reliability. In detail, the DK approach yielded overall SEM values of less than $3^{\circ}$ in the sagittal and frontal planes, and approximately $4^{\circ}$ in the transversal plane. The RMSDp and RMSDw showed comparable, but higher results. These higher values can be explained by the fact that both RMSD parameters include the entire sources of variation, whereas the SEM only represents variation due to pure measurement error. Results for the SEM, RMSDp, and RMSDw for the 3-3-1 IK model were very similar to the DK approach. The 3-1-1-IK model showed smaller values by approximately two degrees in the transversal plane compared to the DK model. This is explained by the fact that the 3-1-1-IK model only had one DoF in the knee joint.

The obtained reliability values compare mostly very well with the findings from recent publications. Kainz et al. [12] compared the same two IK models to the Vicon Plug-in-Gait (PiG) model (which is another standard model in gait analysis) in a population of children with cerebral palsy and typically developing children. Differences between their obtained SEM and our results are below one degree per joint and plane, except for the pelvic and hip sagittal plane in the 3-1-1-IK model and for the hip and knee transversal plane of the PiG compared to our DK model. In the first case, our results showed a SEM of $1.5^{\circ}$ and $1.8^{\circ}$ greater than in the results of Kainz et al. [12], which could be caused by the increased difficulty in pelvic marker placement in the obese participants compared to the lean children. In the second case, our DK model resulted in lower SEM values $\left(1.0^{\circ}\right.$ and $\left.2.0^{\circ}\right)$ compared to the PiG. This might be explained by the lateral thigh and shank markers used by the PiG. Their reliable placement is difficult and errors can easily affect the transversal plane joint kinematics. A recent study gives support to this 


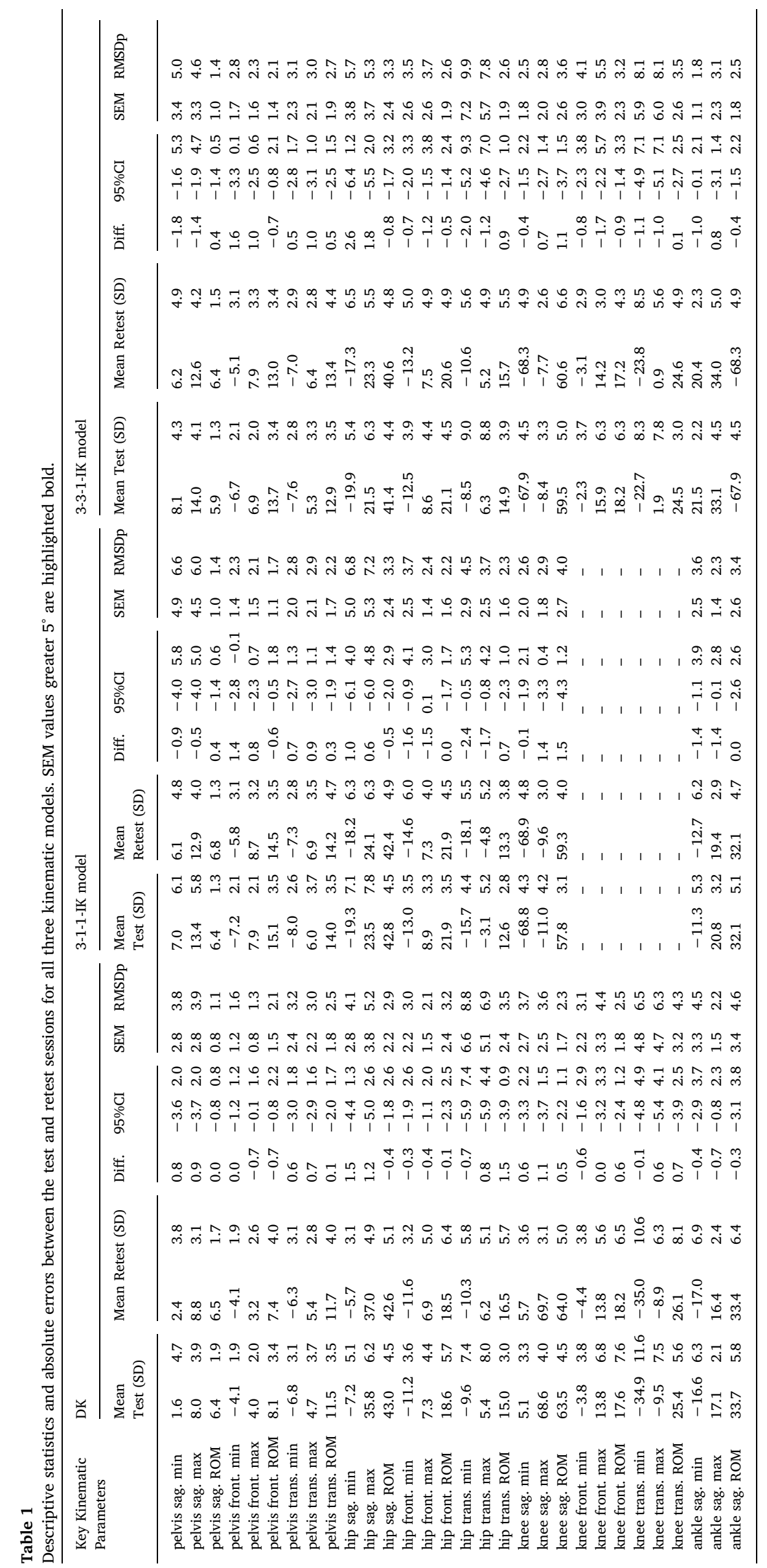



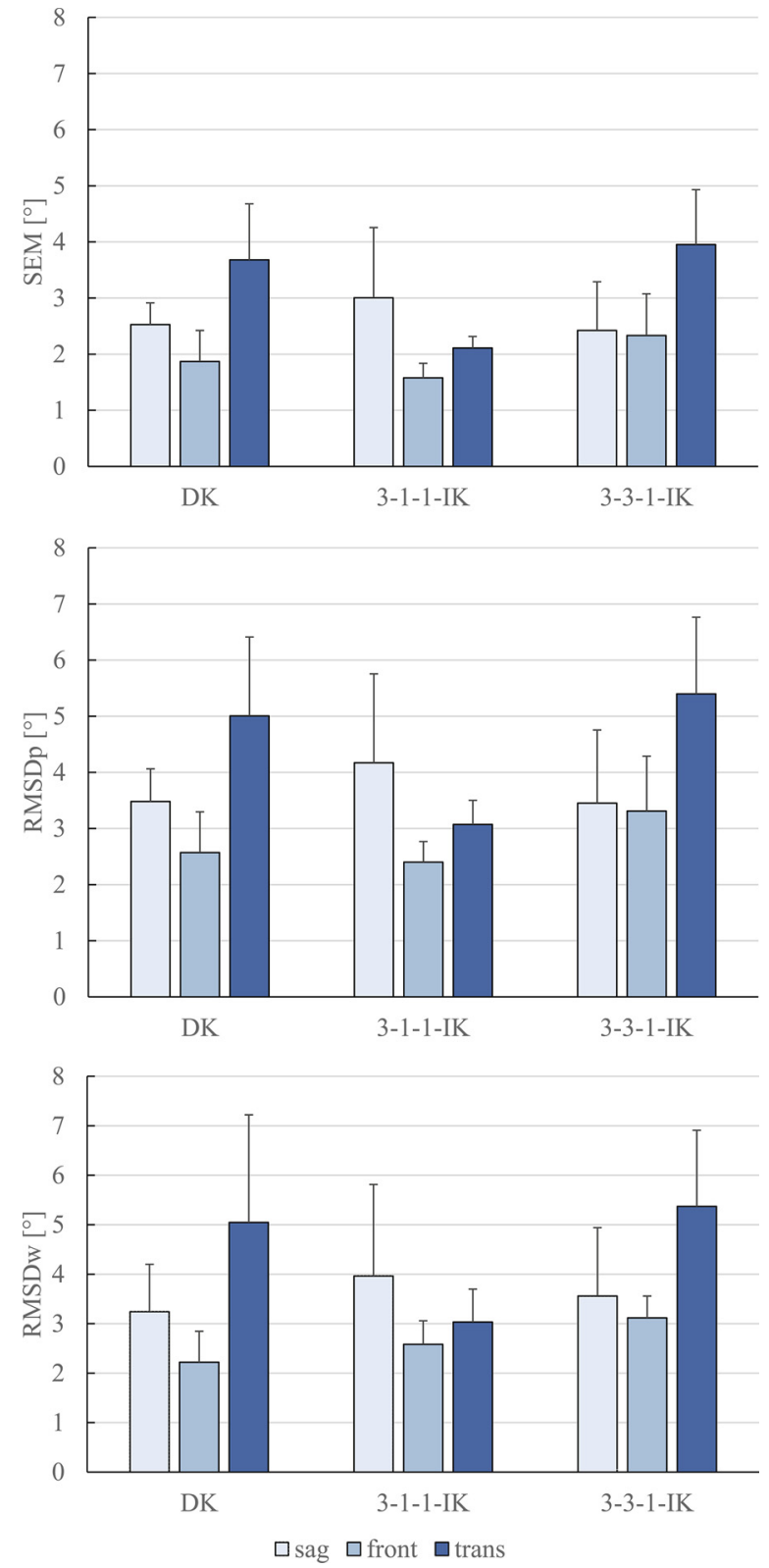

Fig. 2. The standard error of measurement (SEM), the root mean square deviation (RMSDp), both averaged across the three key kinematic parameters, and the root mean square deviation for the entire waveforms (RMSDw) are shown for each anatomical plane (sag, front and trans) and model (DK, 3-1-1-IK and 33-1-IK). The bars represent the mean, the whiskers the observed maximum.

assumption [23]. However, in general, results of both studies are in good agreement to each other and give confidence to the validity of the data. McGinley et al. [8] concluded in their systematic review, that errors between $2^{\circ}$ and $5^{\circ}$ need to be considered during interpretation, but are likely to be reasonable to detect clinically relevant changes in 3D gait analysis. Thus, the obtained results of this study confirm our first hypothesis, that there are no relevant differences in the test-retest reliability between the DK and the IK approach. In line with the margins of error stated by McGinley et al. [8], both of our analyzed IK models showed acceptable reliability. This supports their careful application for MSK-modelling in an overweight or obese population. However, it is noteworthy that presumably only an experienced tester will achieve a similar test-retest reliability in IK models compared to DK models.

The second aim of this study was to assess if the kinematic waveforms from the IK models show any clinical relevant differences compared to the DK approach. This information is important for a more informed interpretation of the MSK IK modelling results, which may deviate from the routine clinical DK modelling results. Observed results give support to our hypothesis as several significant differences were found between both IK models and the DK approach. Most of these differences were continuous offsets, which are in agreement with Kainz et al. [12]. These offsets were likely caused by different model conventions and anatomical segment coordinate frame definitions. The DK model defined the pelvic anatomical reference frame based on the ASIS and sacrum markers. This leads to a pelvic anterior tilt of approximately $10-15^{\circ}$ in the anatomical neutral position. In the IK models, pelvic tilt is 0 degrees in the anatomical neutral position. This offset explains the differences in pelvic tilt and hip flexion/extension kinematics between the DK and IK models. Furthermore, this offset also impacts on pelvic angles in the frontal plane because a floating axis, which is dependent on the original anatomical reference frame, is used to calculate pelvic angles in the frontal plane [1]. The remaining differences were likely caused by variations in joint center locations and joint axes definitions, as well as differences in joint DoF. Differences in hip internal/external rotations were larger in comparison between the 3-1-1-IK and DK model compared to the 3-3-1-IK and DK model, which highlights the impact of different joint constraints. Hence, direct comparison of the IK to DK model outputs should be done with caution and only with considering the differences in the anatomical models [30].

This study has some potential limitations. First, we used a standard marker set with only marginal modifications to the ASIS marker placement to account for soft tissue offset. However, there are recent attempts for obesity specific markers sets [31], which might lead to more accurate and reliable results. Second, different IK and DK models might lead to slightly different reliability results. Third, we could not assess if the DK or IK models were more accurate due to the lack of a gold standard to estimate joint kinematics. Therefore, the results from the comparison of the DK with the IK models should be interpreted with caution. Fourth, we only assessed the reliability of joint kinematics. Reliability of joint kinetics and musculoskeletal estimates (e.g. muscletendon forces) was beyond the scope of this study and should be done in future investigations. Last, it should be noted that we have analyzed both body sides, but only reported the right side in detail. The left side showed comparable reliability patterns, but slightly better values especially for the transversal plane in all models (see supplementary material). This small inconsistency might suggest that the general reported values for the transversal plane might even perform slightly better than currently reported in this paper. However, due to the general problems with soft tissue artifacts in this population, great caution is necessary in interpreting gait analysis data and therefore the more conservative perspective seemed appropriate.

\section{Conclusion}

Our findings showed no differences in the reliability in joint kinematics between our analyzed IK and DK models. Reliability values were clinically acceptable and therefore support the careful use of MSK IK models in overweight or obese populations. Future MSK studies can use our obtained reliability results to judge if an observed difference is caused by a real difference, e.g. due to an intervention, or if an observed difference falls within the error margins of a test-retest scenario. Furthermore, our findings can assist in interpreting kinematic differences between IK and DK models. 

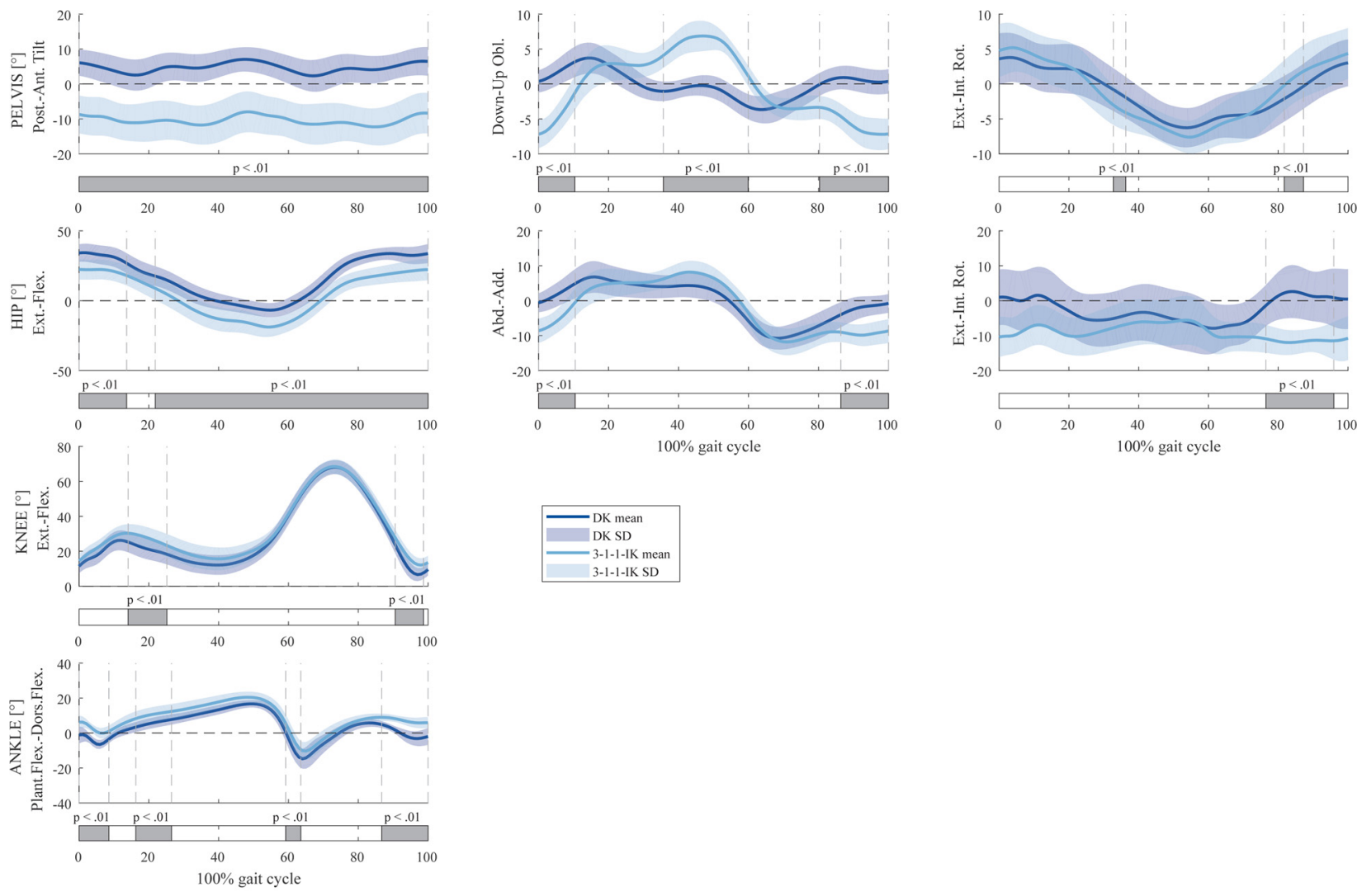

\section{- DK mean}

DK SD

3-1-1-IK SD
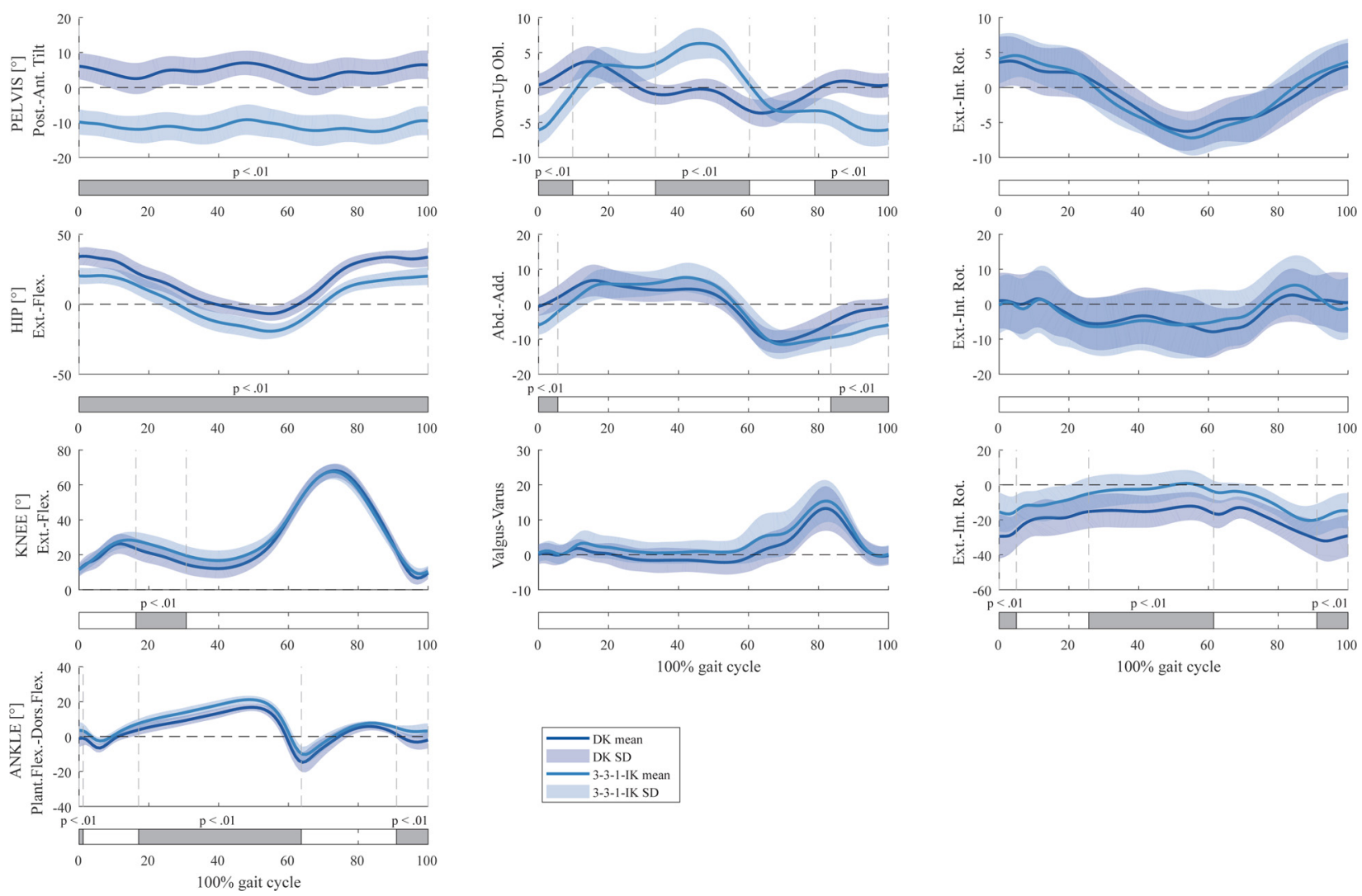

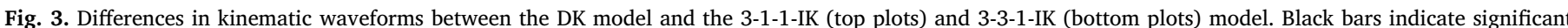
differences between both waveforms, where the SPM $\{\mathrm{t}\}$ values exceeded the Bonferroni corrected alpha level threshold. 


\section{Conflicts of interest}

There is no conflict of interest to declare.

\section{Acknowledgements}

This work was supported by the NFB - Lower Austrian Research and Education Company and the Provincial Government of Lower Austria, Department of Science and Research (grant number LSC13-009). Dr Hans Kainz was funded by a H2020-MSCA individual fellowship. We would like to thank David Artner, MSc. for his support during data recording and Prof. Dr. Susanne Greber-Platzer as well as DDr. Alexandra Kreissl for their great assistance in participant screening and recruitment.

\section{Appendix A. Supplementary data}

Supplementary data associated with this article can be found, in the online version, at https://doi.org/10.1016/j.gaitpost.2018.08.027.

\section{References}

[1] E.S. Grood, W.J. Suntay, A joint coordinate system for the clinical description of three-dimensional motions: application to the knee, J. Biomech. Eng. 105 (1983) 136.

[2] S.L. Delp, F.C. Anderson, A.S. Arnold, P. Loan, A. Habib, C.T. John, E. Guendelman, D.G. Thelen, Opensim: open-source software to create and analyze dynamic simulations of movement, IEEE Trans. Biomed. Eng. 54 (2007) 1940-1950, https://doi. org/10.1109/TBME.2007.901024.

[3] M. Damsgaard, J. Rasmussen, S.T. Christensen, E. Surma, M. de Zee, Analysis of musculoskeletal systems in the anybody modeling system, Simul. Model. Pract. Theory 14 (2006) 1100-1111, https://doi.org/10.1016/j.simpat.2006.09.001.

[4] L. Barber, C. Carty, L. Modenese, J. Walsh, R. Boyd, G. Lichtwark, Medial gastrocnemius and soleus muscle-tendon unit, fascicle, and tendon interaction during walking in children with cerebral palsy, Dev. Med. Child Neurol. 59 (2017) 843-851, https://doi.org/10.1111/dmcn.13427.

[5] D.F. Graham, C.P. Carty, D.G. Lloyd, G.A. Lichtwark, R.S. Barrett, Muscle contributions to recovery from forward loss of balance by stepping, J. Biomech. 47 (2014) 667-674, https://doi.org/10.1016/j.jbiomech.2013.11.047.

[6] M. Wesseling, L.C. Derikx, F. De Groote, W. Bartels, C. Meyer, N. Verdonschot, I. Jonkers, Muscle optimization techniques impact the magnitude of calculated hip joint contact forces, J. Orthop. Res. 33 (2015) 430-438, https://doi.org/10.1002/ jor. 22769.

[7] M.P. Kadaba, H.K. Ramakrishnan, M.E. Wootten, J. Gainey, G. Gorton, G.V.B. Cochran, Repeatability of kinematic, kinetic, and electromyographic data in normal adult gait, J. Orthop. Res. 7 (1989) 849-860.

[8] J.L. McGinley, R. Baker, R. Wolfe, M.E. Morris, The reliability of three-dimensional kinematic gait measurements: a systematic review, Gait Posture 29 (2009) 360-369, https://doi.org/10.1016/j.gaitpost.2008.09.003.

[9] B. Horsak, B. Pobatschnig, A. Baca, S. Greber-Platzer, A. Kreissl, S. Nehrer, B. Wondrasch, R. Crevenna, M. Keilani, A. Kranzl, Within-assessor reliability and minimal detectable change of gait kinematics in a young obese demographic, Gait Posture 54 (2017) 112-118, https://doi.org/10.1016/j.gaitpost.2017.02.028.

[10] S.P. Sankey, R.M.F. Raja Azidin, M.A. Robinson, B. Malfait, K. Deschamps, S. Verschueren, F. Staes, J. Vanrenterghem, How reliable are knee kinematics and kinetics during side-cutting manoeuvres? Gait Posture 41 (2015) 905-911, https:// doi.org/10.1016/j.gaitpost.2015.03.014.

[11] D.L. Mason, S.J. Preece, C.A. Bramah, L.C. Herrington, Reproducibility of kinematic measures of the thoracic spine, lumbar spine and pelvis during fast running, Gait Posture 43 (2016) 96-100, https://doi.org/10.1016/j.gaitpost.2013.11.007.

[12] H. Kainz, D. Graham, J. Edwards, H.P.J. Walsh, S. Maine, R.N. Boyd, D.G. Lloyd,
L. Modenese, C.P. Carty, Reliability of four models for clinical gait analysis, Gait Posture 54 (May) (2017) 325-331, https://doi.org/10.1016/j.gaitpost.2017.04. 001.

[13] Z.F. Lerner, W.J. Board, R.C. Browning, Effects of obesity on lower extremity muscle function during walking at two speeds, Gait Posture 39 (2014) 978-984, https:// doi.org/10.1016/j.gaitpost.2013.12.020.

[14] Z.F. Lerner, W.J. Board, R.C. Browning, Pediatric obesity and walking duration increase medial tibiofemoral compartment contact forces, J. Orthop. Res. 34 (2016) 97-105, https://doi.org/10.1002/jor.23028.

[15] D.J. Haight, Z.F. Lerner, W.J. Board, R.C. Browning, A comparison of slow, uphill and fast, level walking on lower extremity biomechanics and tibiofemoral joint loading in obese and nonobese adults, J. Orthop. Res. 32 (2014) 324-330, https:// doi.org/10.1002/jor.22497.

[16] A. Peters, B. Galna, M. Sangeux, M. Morris, R. Baker, Quantification of soft tissue artifact in lower limb human motion analysis: a systematic review, Gait Posture 31 (2010) 1-8, https://doi.org/10.1016/j.gaitpost.2009.09.004.

[17] T.-W. Lu, J.J. O'connor, Bone position estimation from skin marker co-ordinates using global optimisation with joint constraints, J. Biomech. 32 (1999) 129-134.

[18] M.S. Andersen, M. Damsgaard, J. Rasmussen, Kinematic analysis of over-determinate biomechanical systems, Comput. Methods Biomech. Biomed. Eng. 12 (2009) 371-384, https://doi.org/10.1080/10255840802459412.

[19] H. Kainz, L. Modenese, D.G. Lloyd, S. Maine, H.P.J. Walsh, C.P. Carty, Joint kinematic calculation based on clinical direct kinematic versus inverse kinematic gait models, J. Biomech. 49 (2016) 1658-1669, https://doi.org/10.1016/j.jbiomech. 2016.03.052.

[20] D.A. Winter, Biomechanics and Motor Control of Human Movement, third edition, John Wiley \& Sons, Waterloo, 2005.

[21] M. Pospischill, A. Kranzl, B. Attwenger, K. Knahr, Minimally invasive compared with traditional transgluteal approach for total hip arthroplasty: a comparative gait analysis, J. Bone Joint Surg. Am. 92 (2010) 328-337, https://doi.org/10.2106/ JBJS.H.01086.

[22] G.T. Mindler, A. Kranzl, C.A.M. Lipkowski, R. Ganger, C. Radler, Results of gait analysis including the Oxford foot model in children with clubfoot treated with the Ponseti method, J. Bone Joint Surg. Am. 96 (2014) 1593-1599, https://doi.org/10. 2106/JBJS.M.01603.

[23] B. Svoboda, A. Kranzl, A study of the reproducibility of the marker application of the Cleveland Clinic Marker Set including the Plug-In Gait Upper Body Model in clinical gait analysis, Gait Posture 36 (2012) S62-S63, https://doi.org/10.1016/j. gaitpost.2011.10.286.

[24] R.B. Davis, S. Õunpuu, D. Tyburski, J.R. Gage, A gait analysis data collection and reduction technique, Hum. Mov. Sci. 10 (1991) 575-587, https://doi.org/10.1016/ 0167-9457(91)90046-Z.

[25] G. Wu, S. Siegler, P. Allard, C. Kirtley, A. Leardini, D. Rosenbaum, M. Whittle, D.D. D'Lima, L. Cristofolini, H. Witte, O. Schmid, I. Stokes, Standardization and Terminology Committee of the International Society of Biomechanics, ISB recommendation on definitions of joint coordinate system of various joints for the reporting of human joint motion-part I: ankle, hip, and spine, Int. Soc. Biomechan. J. Biomech. 35 (2002) 543-548.

[26] S.L. Delp, J.P. Loan, M.G. Hoy, F.E. Zajac, E.L. Topp, J.M. Rosen, An interactive graphics-based model of the lower extremity to study orthopaedic surgical procedures, IEEE Trans. Biomed. Eng. 37 (1990) 757-767, https://doi.org/10.1109/10. 102791.

[27] H. Kainz, H.X. Hoang, C. Stockton, R.R. Boyd, D.G. Lloyd, C.P. Carty, Accuracy and reliability of marker-based approaches to scale the pelvis, thigh, and shank segments in musculoskeletal models, J. Appl. Biomech. 33 (2017) 354-360, https:// doi.org/10.1123/jab.2016-0282.

[28] W. Vincent, J.P. Weir, Statistics in Kinesiology, 4th ed., Human Kinetics, 2012.

[29] B. Horsak, C. Schwab, C. Clemens, A. Baca, S. Greber-Platzer, A. Kreissl, A. Kranzl, Is the reliability of 3D kinematics of young obese participants dependent on the hip joint center localization method used? Gait Posture 59 (2018) 65-70, https://doi. org/10.1016/j.gaitpost.2017.09.029.

[30] T.C. Pataky, One-dimensional statistical parametric mapping in Python, Comput Methods Biomech. Biomed. Eng. 15 (2012) 295-301, https://doi.org/10.1080/ 10255842.2010.527837.

[31] Z.F. Lerner, W.J. Board, R.C. Browning, Effects of an obesity-specific marker set on estimated muscle and joint forces in walking, Med. Sci. Sports Exercise 46 (2014) 1261-1267, https://doi.org/10.1249/MSS.0000000000000218. 